OPEN ACCESS

Edited by:

Kai He,

Lanzhou University, China

Reviewed by:

Dai-Yin Chao,

Shanghai Institutes for Biological

Sciences (CAS), China

Caiji Gao,

South China Normal University, China

*Correspondence:

Ren-Jie Tang

ritang@berkeley.edu

Sheng Luan

sluan@berkeley.edu

Specialty section: This article was submitted to Plant Traffic and Transport,

a section of the journal

Frontiers in Plant Science

Received: 04 January 2018

Accepted: 16 February 2018

Published: 12 March 2018

Citation:

Yan Y-W, Mao D-D, Yang L, Qi J-L, Zhang $X-X$, Tang $Q-L, L i Y-P$,

Tang R-J and Luan S (2018)

Magnesium Transporter MGT6 Plays

an Essential Role in Maintaining

Magnesium Homeostasis and Regulating High Magnesium

Tolerance in Arabidopsis.

Front. Plant Sci. 9:274

doi: 10.3389/fpls.2018.00274

\section{Magnesium Transporter MGT6 Plays an Essential Role in Maintaining Magnesium Homeostasis and Regulating High Magnesium Tolerance in Arabidopsis}

\author{
Yu-Wei Yan1,2, Dan-Dan Mao 3,4, Lei Yang ${ }^{3}$, Jin-Liang Qi1,3, Xin-Xin Zhang,,5, \\ Qing-Lin Tang ${ }^{1,6}$, Yang-Ping Li ${ }^{1,2}$, Ren-Jie Tang ${ }^{1 *}$ and Sheng Luan ${ }^{1 *}$
}

'Department of Plant and Microbial Biology, University of California, Berkeley, Berkeley, CA, United States, ${ }^{2}$ Key Laboratory of Biology and Genetic Improvement of Maize in Southwest Region, Maize Research Institute of Sichuan Agricultural University, Chengdu, China, ${ }^{3}$ Nanjing University-Nanjing Forestry University Joint Institute for Plant Molecular Biology, State Key Laboratory for Pharmaceutical Biotechnology, College of Life Sciences, Nanjing University, Nanjing, China, ${ }^{4}$ College of Life Sciences, Hunan Normal University, Changsha, China, ${ }^{5}$ Key Laboratory of Saline-Alkali Vegetation Ecology Restoration in Oil Field, Ministry of Education, Alkali Soil Natural Environmental Science Center, Northeast Forestry University, Harbin, China, ${ }^{6}$ Key Laboratory of Horticulture Science for Southern Mountainous Regions, Southwest University, Chongqing, China

Magnesium (Mg) is one of the essential nutrients for all living organisms. Plants acquire $\mathrm{Mg}$ from the environment and distribute within their bodies in the ionic form via $\mathrm{Mg}^{2+}$ permeable transporters. In Arabidopsis, the plasma membrane-localized magnesium transporter MGT6 mediates $\mathrm{Mg}^{2+}$ uptake under Mg-limited conditions, and therefore is important for the plant adaptation to low-Mg environment. In this study, we further assessed the physiological function of MGT6 using a knockout T-DNA insertional mutant allele. We found that MGT6 was required for normal plant growth during various developmental stages when the environmental $\mathrm{Mg}^{2+}$ was low. Interestingly, in addition to the hypersensitivity to $\mathrm{Mg}^{2+}$ limitation, mgt6 mutants displayed dramatic growth defects when external $\mathrm{Mg}^{2+}$ was in excess. Compared with wild-type plants, mgt6 mutants generally contained less $\mathrm{Mg}^{2+}$ under both low and high external $\mathrm{Mg}^{2+}$ conditions. Reciprocal grafting experiments further underpinned a role of MGT6 in a shoot-based mechanism for detoxifying excessive $\mathrm{Mg}^{2+}$ in the environment. Moreover, we found that mgt6 mgt7 double mutant showed more severe phenotypes compared with single mutants under both low- and high- $\mathrm{Mg}^{2+}$ stress conditions, suggesting that these two MGT-type transporters play an additive role in controlling plant $\mathrm{Mg}^{2+}$ homeostasis under a wide range of external $\mathrm{Mg}^{2+}$ concentrations.

Keywords: $\mathrm{Mg}^{2+}$ transporter, $\mathrm{Mg}^{2+}$ homeostasis, Arabidopsis, MGT6, MGT7

\section{INTRODUCTION}

Magnesium $(\mathrm{Mg})$ is an essential macronutrient for plants. Being the most abundant free divalent cation in living cells, $\mathrm{Mg}^{2+}$ serves as a counter ion for nucleotides and a central metal for chlorophylls, and acts as a cofactor for many enzymes in catalytic processes. $\mathrm{Mg}^{2+}$ also contributes to membrane stabilization and active conformation of macromolecules (Shaul, 2002). 
Both low and high levels of $\mathrm{Mg}$ present in the soil are deleterious to plant growth, thus affecting crop production. Due to unbalanced application of chemical fertilizers, plants may exhibit Mg deficiency symptoms in the presence of high levels of other cations such as calcium $\left(\mathrm{Ca}^{2+}\right)$ and potassium $\left(\mathrm{K}^{+}\right)$in the soil (Hermans et al., 2013). Moreover, excessive aluminum $\left(\mathrm{Al}^{3+}\right)$ in acidic soils or other heavy metals severely inhibit the uptake of $\mathrm{Mg}^{2+}$, resulting in $\mathrm{Mg}$ deficiency in the plants. These problems lead to reduction in crop yield as well as higher susceptibility to some plant diseases. On the other hand, high levels of $\mathrm{Mg}$ are found in serpentine soils featuring a low $\mathrm{Ca} / \mathrm{Mg}$ ratio (Brady et al., 2005). Genome sequencing of Arabidopsis lyrata plants grown in serpentine or non-serpentine habitats has identified a number of polymorphisms associated with $\mathrm{Ca}^{2+}$ and $\mathrm{Mg}^{2+}$ transport (Turner et al., 2010). Although it is critical for plant cells to maintain an optimal $\mathrm{Mg}^{2+}$ level for normal growth and development, the transport and regulatory mechanisms that govern $\mathrm{Mg}^{2+}$ acquisition, distribution, and reallocation are poorly understood (Tang and Luan, 2017).

In bacterial cells, there are at least three distinct types of membrane proteins CorA, MgtE, and MgtA/B that are capable of transporting $\mathrm{Mg}^{2+}$. While the MgtE channel and the P-type ATPases MgtA/B do not seem to have any close homologs in plants, there is a major family of $\mathrm{Mg}^{2+}$ transporters (MGTs) related to bacterial CorA proteins (Li et al., 2001). They are also named as "MRS2s" based on the ability to rescue the yeast mrs2 mutant lacking the Mrs2 protein, a yeast homolog of CorA-type transporter that mediates $\mathrm{Mg}^{2+}$ transport into the mitochondrial matrix (Schock et al., 2000). The CorA-family proteins feature a unique topology with two closely spaced, C-terminal transmembrane (TM) domains, the first of which contains a conserved GMN (Gly-Met-Asn) tripeptide motif that is essential for $\mathrm{Mg}^{2+}$ transport (Szegedy and Maguire, 1999). Crystal structure of the Thermotoga maritima CorA establishes the protein as a pentameric cone-shaped ion channel (Eshaghi et al., 2006; Lunin et al., 2006).

Several members of the Arabidopsis MGTs facilitate $\mathrm{Mg}^{2+}$ transport in bacteria or yeast (Li et al., 2001, 2008; Mao et al., 2008, 2014; Gebert et al., 2009). Genes coding for MGT-type transporters are widely expressed in various plant tissues and cell types in Arabidopsis (Li et al., 2001; Gebert et al., 2009) and the proteins are targeted to plasma membrane or intracellular membranes, implicating MGT members functioning in $\mathrm{Mg}^{2+}$ transport across multiple cellular membranes. MGT1 is mainly expressed in the root hair and the elongation zone as well as the vascular tissues and leaf trichomes (Gebert et al., 2009), suggesting a potential role in $\mathrm{Mg}^{2+}$ translocation in these particular cell types. MGT2 and MGT3 are associated with vacuolar membrane and possibly involved in $\mathrm{Mg}^{2+}$ homeostasis in leaf mesophyll cells (Conn et al., 2011). Quite a few MGTs including MGT4, MGT5, and MGT9 are highly expressed in pollen and anther cells, and are required for plant reproduction, suggesting that active $\mathrm{Mg}^{2+}$ transport is critical for pollen development (Li et al., 2008, 2015; Chen et al., 2009; Xu et al., 2015). MGT10 is localized in the chloroplast envelope, and is strongly expressed in the rosette and cauline leaves, indicating its possible function in $\mathrm{Mg}^{2+}$ translocation into chloroplasts
(Drummond et al., 2006). Indeed, two recent studies confirmed that mutant plants lacking MGT10 show defects in chloroplast development and plant photosynthesis (Liang et al., 2017; Sun et al., 2017). In rice, OsMGT1 is localized to the plasma membrane and its rapid up-regulation upon $\mathrm{Al}^{3+}$ stress confers $\mathrm{Al}^{3+}$ tolerance on rice plants as a result of enhanced $\mathrm{Mg}^{2+}$ uptake (Chen et al., 2012). Interestingly, OsMGT1 plays a role in rice salt tolerance possibly through activating the transport activity of OsHKT1;5 (Chen et al., 2017).

Among all the MGT-type $\mathrm{Mg}^{2+}$ transporters in Arabidopsis, MGT6 and MGT7 are thought to be more directly involved in controlling cellular $\mathrm{Mg}^{2+}$ homeostasis because impairment of MGT6 or MGT7 function renders Arabidopsis plants hypersensitive to low-Mg conditions (Gebert et al., 2009; Mao et al., 2014; Oda et al., 2016). MGT6 appears to be localized to the plasma membrane and mediate the high-affinity $\mathrm{Mg}^{2+}$ uptake via roots (Mao et al., 2014). Consistent with this role, expression of MGT6 is dramatically up-regulated at the transcriptional level when external $\mathrm{Mg}^{2+}$ becomes limited (Mao et al., 2014). MGT7 is preferentially expressed in roots, and also plays an important role for plant adaptation to low-Mg conditions although the mechanism is not clear (Gebert et al., 2009). In this study, we showed that MGT6 is equally important for controlling plant $\mathrm{Mg}^{2+}$ homeostasis under normal and high-Mg conditions. We uncovered a shoot-based mechanism that underlies MGT6 function in detoxifying excessive $\mathrm{Mg}^{2+}$, in addition to its role in root $\mathrm{Mg}^{2+}$ uptake under Mg-limited conditions. Furthermore, by analyzing the mgt6 mgt7 double mutant, we showed that these two $\mathrm{Mg}^{2+}$ transporters MGT6 and MGT7 play an overlapping role in maintaining essential $\mathrm{Mg}^{2+}$ homeostasis under a wide range of external $\mathrm{Mg}^{2+}$ concentrations.

\section{MATERIALS AND METHODS}

\section{Plant Materials and Growth Conditions}

Arabidopsis thaliana ecotype Col-0 was used in this study. T-DNA insertional mutant lines were obtained from the Arabidopsis Biological Resource Center. The seed stock IDs are as follows: SALK_205483 (mgt6) and SALK_064741 (mgt7). The double mutant $m g t 6$ mgt7 was generated by crossing $m g t 7$ to $m g t 6$ mutant, and progeny of F2 generation was screened for double homozygous mutations in MGT6 and MGT7 using a PCR-based genotyping approach.

Wild-type and mutant plants were grown in the soil at $22^{\circ} \mathrm{C}$ under the 16-h-light/8-h-dark condition in the greenhouse. Hydroponically grown plants were generally kept in the $1 / 6$ strength MS solution under the short-day condition (8-hlight/16-h-dark) in the greenhouse. Fresh liquid solutions were replaced twice a week.

\section{Phenotypic Assays}

Arabidopsis seeds of different genotypes were sterilized with 10\% bleach for $5 \mathrm{~min}$ and washed in sterilized water for 3 times. Seeds were sown on the solid plates supplemented with different concentrations of $\mathrm{Mg}^{2+}$. The basal medium contained 1/6 strength of MS salt (Murashige and Skoog, 1962) in which $\mathrm{MgSO}_{4}$ 
was replaced by the $\mathrm{K}_{2} \mathrm{SO}_{4}$. Different concentrations of $\mathrm{MgCl}_{2}$ were added as the $\mathrm{Mg}^{2+}$ source. After 2-day stratification at $4^{\circ} \mathrm{C}$, plates were vertically grown at $22^{\circ} \mathrm{C}$ in the growth chamber.

For the post-germination assay, seeds were first sown on MS medium solidified with $1 \%$ phytoagar. After germination, 5-dayold seedlings were transferred onto $1 / 6 \mathrm{Mg}^{2+}$-free MS medium (containing $1 \%$ sucrose, $\mathrm{pH}=5.8$, solidified with $0.8 \%$ agarose) supplemented with $\mathrm{Mg}^{2+}$ at the indicated concentrations.

For phenotypic assay in the hydroponics, 7-day-old seedlings were transferred to liquid solutions containing 1/6 MS salts supplemented with $1.25 \mathrm{mM} \mathrm{MgSO}_{4}$. After 2-week culture, the plants were treated with solutions containing different concentrations of $\mathrm{Mg}^{2+}$.

\section{Functional Complementation}

For complementation of the mgt6 mutant, a $3.5-\mathrm{kb}$ genomic fragment including the MGT6 coding region as well as $1.5 \mathrm{~kb}$ of the $5^{\prime}$ flanking DNA upstream of the starting codon was amplified by PCR from Arabidopsis genomic DNA with forward (5'-AC GGATAAATGTGGGGATGCTTG-3 $\left.3^{\prime}\right)$ and reverse $\left(5^{\prime}\right.$-CCAA ATCAAATCAACCCATAAAC- $3^{\prime}$ ) primers. The PCR product was cloned into the SmaI site of the binary vector pCAMBIA1300. After sequencing, the construct was transformed into Agrobacterium tumefaciens strain GV3101 and introduced into mgt6 mutant plants by the floral dip method (Clough and Bent, 1998). Transgenic seeds were screened on MS medium supplemented with $25 \mathrm{mg} / \mathrm{L}$ hygromycin. Resistant seedlings were transplanted to soil and grown in the greenhouse for seed propagation. T3 homozygous transgenic plants were subject to gene expression analysis and phenotypic assays together with wild-type plants and mgt6 mutants.

\section{RNA Isolation and Gene Expression Analysis}

Total RNA was extracted from plant materials using the TRIzol reagent (Invitrogen). After being digested by DNase I (Invitrogen) to decontaminate DNA, cDNA was generated from RNA samples at $42^{\circ} \mathrm{C}$ using SuperScript II reverse transcriptase (Invitrogen). The resultant cDNA samples were used for PCR amplification with the gene-specific primers. Quantitative real-time PCR was performed on the DNA Engine Opticon System (MJ Research) using the SYBR Green Realtime PCR Master Mix to monitor double-stranded DNA products. Data were calculated based on the comparative threshold cycle method. The relative expression of each $\mathrm{Mg}$ starvation marker gene was double-normalized using the housekeeping gene ACTIN2 and using the control expression values measured in the wild type when external $\mathrm{Mg}^{2+}$ is $1.5 \mathrm{mM}$.

\section{Grafting Experiments}

Reciprocal grafting experiments were performed as previously described with minor modifications (Marsch-Martínez et al., 2013). Seeds were sown on MS medium containing $1 \%$ agar and $2 \%$ sucrose, and grown vertically in the growth chamber $\left(22^{\circ} \mathrm{C}, 14\right.$-h-light/10-h-dark $)$ after 2-day stratification at $4^{\circ} \mathrm{C}$. Six-day-old Arabidopsis seedlings were transversely cut with a sharp blade in the middle position of the hypocotyl so that each individual seedling was divided into two parts. Subsequently, different parts of each material were re-assembled and grafted on half MS medium supplemented with $1.2 \%$ agar, $0.5 \%$ sucrose, $3 \mathrm{mg} / \mathrm{L}$ Benomyl [methyl 1-(butylcarbamoyl)-2-benzimidazolecarbamate], $0.02 \mathrm{mg} / \mathrm{L}$ IAA (indole acetic acid) and $0.04 \mathrm{mg} / \mathrm{L}$ 6-BA (6-benzylaminopurine). The grafted seedlings were grown vertically in the growth chamber for another 10 days to allow the formation of the graft union. Successfully unified seedlings with the same size and status were then transferred to the hydroponic culture for further experiments.

\section{Measurement of the $\mathrm{Mg}$ and $\mathrm{Ca}$ Content}

Plant samples were harvested from root and shoot tissues, respectively, and briefly washed with $\mathrm{ddH}_{2} \mathrm{O}$ for $10 \mathrm{~s}$. The samples were then thoroughly dried up in the oven at $80^{\circ} \mathrm{C}$. The dry matters were collected in the $15 \mathrm{~mL}$ centrifuge tubes (ions free) and digested with $1 \mathrm{~mL}$ ultrapure $\mathrm{HNO}_{3}$ (Sigma-Aldrich) in the water bath at $95^{\circ} \mathrm{C}$ for $4 \mathrm{~h}$. Digested samples were diluted to the appropriate concentrations with $\mathrm{ddH}_{2} \mathrm{O}$, and the elemental concentrations were determined by inductively coupled plasma optical emission spectroscopy (ICP-OES; PerkinElmer, Waltham, MA, United States).

\section{RESULTS}

\section{Knockout Mutation in MGT6 Leads to Plant Hypersensitivity to Mg Deficiency}

In a previous study, we have shown that knock-down of MGT6 in transgenic plants by RNA interference resulted in growth retardation under low-Mg conditions (Mao et al., 2014). To further address the physiological role of MGT6, we isolated a previously unidentified T-DNA insertional mutant from the SALK collection (SALK_203866), in which the T-DNA insertion is located in the third exon of MGT6, 39 base pair (bp) upstream of the stop codon (Figure 1A). RT-PCR analyses showed that full-length MGT6 transcript was not detectable in the mgt6 mutant, while MGT4 gene located in the same chromosome is normally expressed (Figure 1B). Consistent with earlier findings, mutation in MGT6 leads to hypersensitivity to $\mathrm{Mg}$ limitation in that the mgt6 mutants experienced growth defects at the germination stage (Figures 1C-F). When germinated on the medium containing no $\mathrm{Mg}^{2+}$ or $0.01 \mathrm{mM} \mathrm{Mg}^{2+}$, the $m g t 6$ mutants showed shorter roots and smaller and pale cotyledons (Figures 1C,D). In the presence of $0.25 \mathrm{mM} \mathrm{Mg}^{2+}$, mgt6 seedlings appeared more normal, albeit still smaller than the wild-type (Figure 1E). Early seedling establishment during germination became comparable between wild-type and mutant plants when external $\mathrm{Mg}^{2+}$ reached $1.5 \mathrm{mM}$ (Figure 1F). Statistical analysis of root length (Figure 1G) and seedling fresh weight (Figure $\mathbf{1 H}$ ) verified the hypersensitivity to $\mathrm{Mg}$ deficiency in the mgt6 mutant. 
A

MGT6 (AT3G58970)

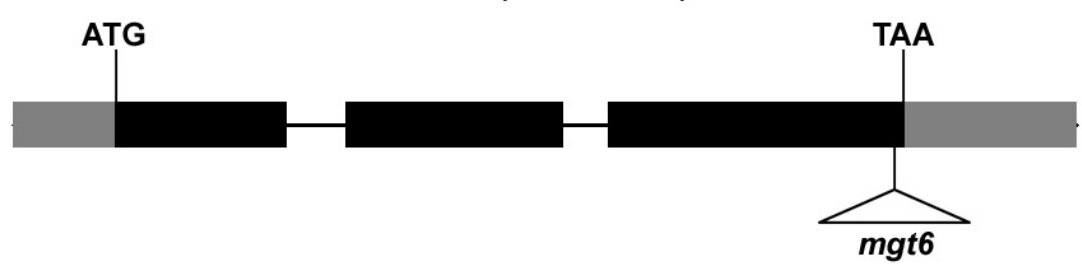

B

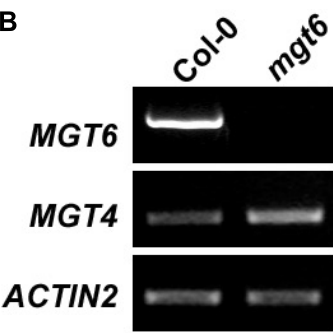

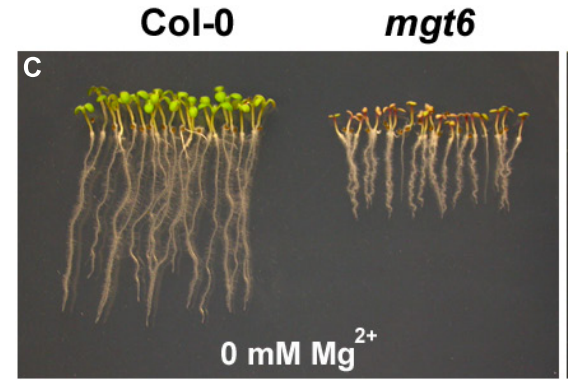

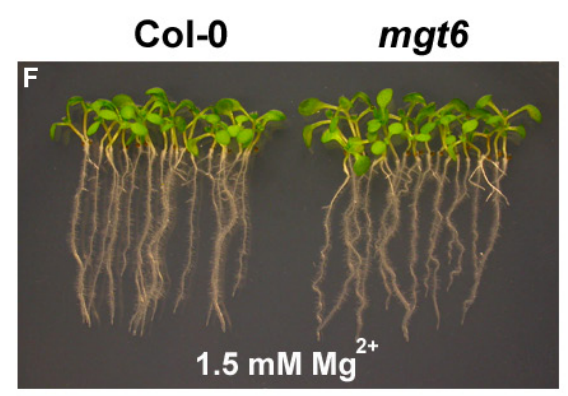

Col-0
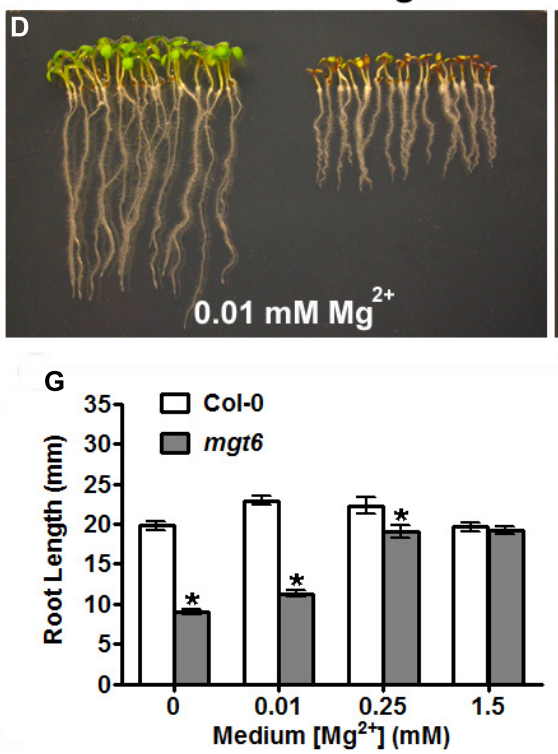

Col-0
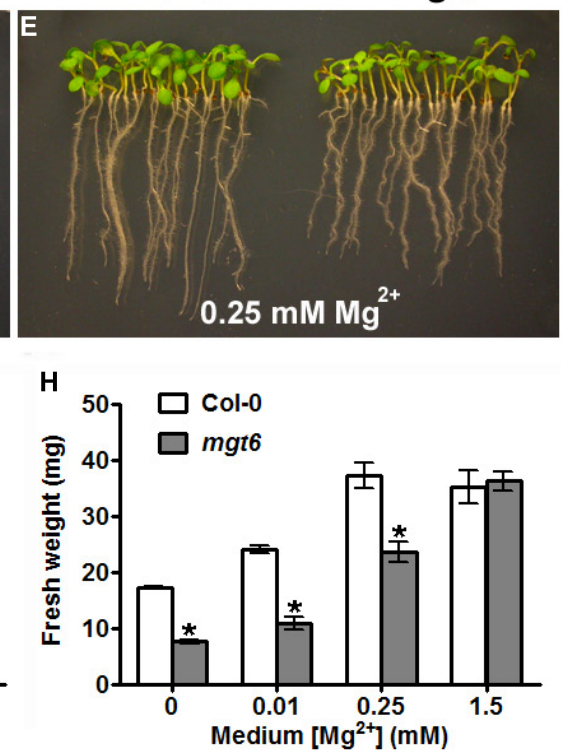

FIGURE 1 | The T-DNA insertional mgt6 mutant is hypersensitive to $\mathrm{Mg}^{2+}$ deficiency. (A) Schematic diagram of the T-DNA insertion in the mgt6 mutant. Exons and introns are depicted to scale by boxes and lines, respectively. The coding region of the gene is shown as black boxes while the $5^{\prime}$ and $3^{\prime}$ UTR of the cDNA is shown as light-shaded boxes. The position of the T-DNA is indicated by the triangle. (B) RT-PCR analysis of MGT6 and MGT4 in wild-type and mgt6 seedlings.

(C-F) Growth phenotype of young seedlings 6 days after seed germination on the medium supplemented with different concentrations of Mg ${ }^{2+}$. (G) Quantification of root length of 7-day-old seedlings. (H) Quantification of seedling fresh weight. Data represent means \pm SE of four replicate experiments. Asterisks indicate significant difference between the wild type and mgt6 mutant (Student's $t$-test, ${ }^{*} P<0.05$ ).

Because MGT-type transporters are capable of transporting several divalent cations in bacteria and yeast ( $\mathrm{Li}$ et al., 2001; Mao et al., 2008), we examined the growth of mgt6 mutant in the absence of other divalent cation nutrients. Whereas mgt6 consistently displayed growth defects in the absence of $\mathrm{Mg}^{2+}$, seedling growth appeared indistinguishable between wild type and $m g t 6$ on the medium lacking other divalent cations including $\mathrm{Ca}^{2+}, \mathrm{Fe}^{2+}, \mathrm{Mn}^{2+}$, and $\mathrm{Zn}^{2+}$ (Supplementary Figure S1). These data suggest that under physiological conditions MGT6 may function in plants to cope with variable external Mg status, but is not relevant to other divalent cations.

\section{MGT6 Is Required for Plant Growth in Arabidopsis Under a Wide Range of External $\mathbf{M g}^{2+}$ Concentrations}

To extend the phenotypic analysis of the mgt6 mutant, we grew the seedlings of the mutant together with the wildtype plants on the plates containing various levels of $\mathrm{Mg}^{2+}$ in the post-germination assay. When grown on the low$\mathrm{Mg}$ medium containing $0,0.01,0.05$, or $0.25 \mathrm{mM} \mathrm{Mg}^{2+}$, the mgt6 mutant plants were clearly stunted as compared with Col-0 (Figure 2A); the primary roots were shorter (Figure 2B) and the seedling fresh weight was significantly reduced (Figure 2C). Because $\mathrm{Mg}^{2+}$ is the central structural cation for chlorophyll, we analyzed the chlorophyll content in the young leaves and found that the mutant had a lower chlorophyll level under extremely low-Mg conditions $(0,0.01$, and $0.05 \mathrm{mM} \mathrm{Mg}^{2+}$; Figure 2D). When the medium $\mathrm{Mg}^{2+}$ levels reached a moderate range $(0.75,1.25$, and $3 \mathrm{mM})$, the growth of mgt6 mutants appeared comparable to that of wild-type (Figure 2A), although primary root length or seedling fresh weight was slightly affected (Figures 2B,C). Notably, in the presence of $6 \mathrm{mM} \mathrm{Mg}^{2+}$ that is regarded as high, the mgt6 seedlings exhibited a strong growth defect (Figure 2A), with much lower fresh weight (Figure 2C) and reduced chlorophyll content (Figure 2D) than wild-type plants. These data suggested that the mgt6 mutant is not only 


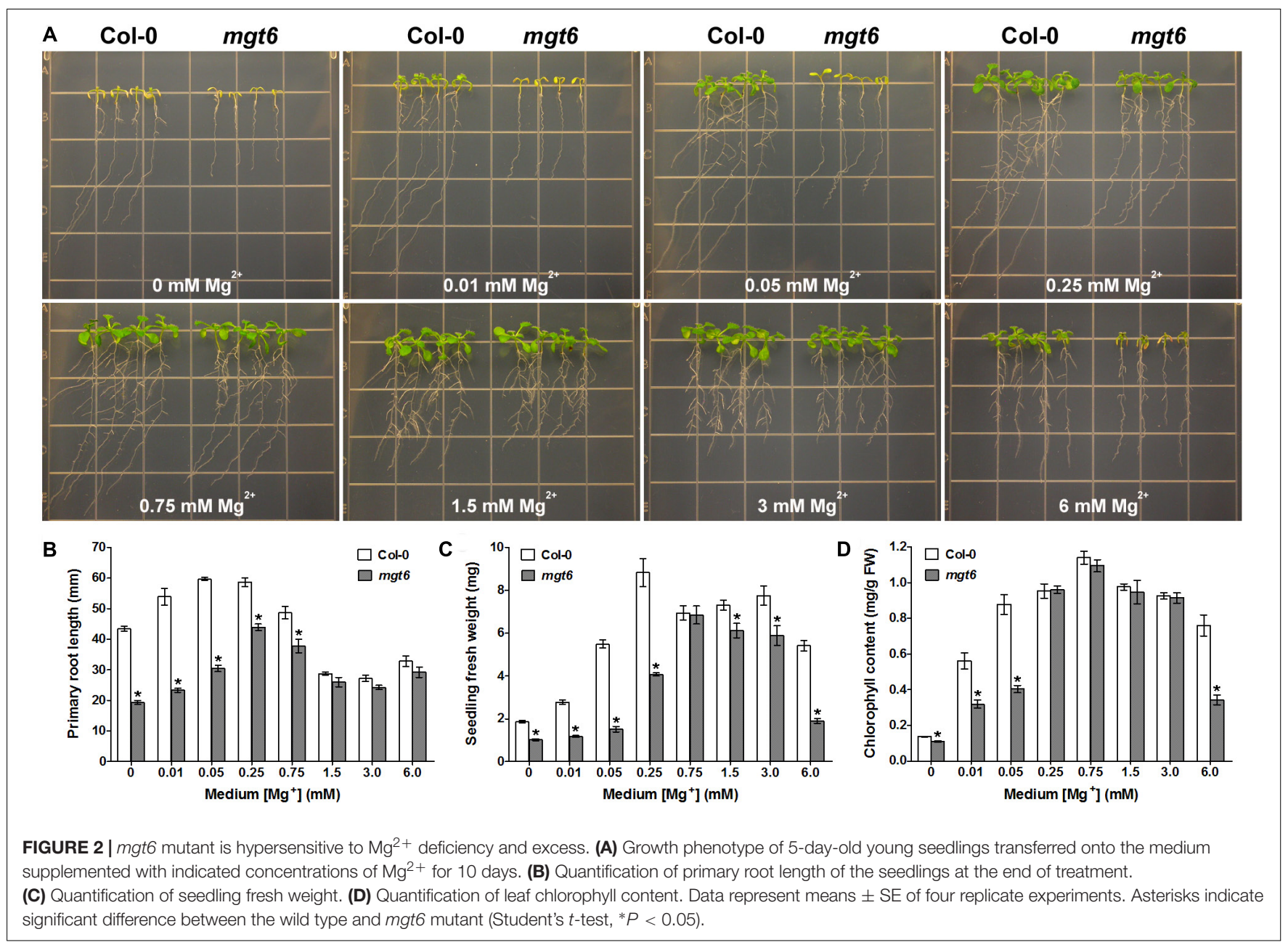

compromised under low-Mg levels but also hypersensitive to high-Mg stress.

To verify the observed phenotypes in the mgt6 mutant resulted from MGT6 mutation, we conducted a complementation test. A genomic fragment of MGT6 was introduced into the mgt6 mutant. Several homozygous transgenic lines with a similar MGT6 transcript level to that in wild type were obtained (Supplementary Figure S2B). Phenotypic analysis of two representative lines showed that seedling growth defects were fully rescued under both low- and high-Mg conditions (Supplementary Figure S2), suggesting MGT6 is indeed required for plant adaptation to $\mathrm{Mg}$ deficiency as well as plant tolerance to high-Mg stress.

To assess the function of MGT6 in mature plants, we grew wild-type and mgt6 plants to flowering stage in the hydroponic solutions with defined levels of external $\mathrm{Mg}^{2+}$. We found that the mgt6 plants showed compromised growth in all conditions tested (Figure $\mathbf{3 A}$ ), but the growth difference was much more pronounced between wild-type and mgt6 plants under extremely low (0.01 and $0.05 \mathrm{mM})$ and high- $\mathrm{Mg}^{2+}$ $(10 \mathrm{mM})$ conditions, as revealed by the root and shoot biomass (Figures 3B,C). These results suggest that MGT6 is essential for plant growth at all developmental stages under a wide range of
$\mathrm{Mg}^{2+}$ concentrations in the environment, and particularly plays an important role in plant adaption to low- and high- $\mathrm{Mg}^{2+}$ stresses.

\section{MGT6 Controls Plant $\mathrm{Mg}^{2+}$ Homeostasis in Both Root and Shoot Tissues}

In order to investigate how plant $\mathrm{Mg}^{2+}$ homeostasis is affected by loss of MGT6 function under various conditions, we measured metal content in the roots and shoots of the wild type and mgt6. We first employed the plant materials cultivated in vitro after 2 weeks' growth on the plates. As expected, compared with wildtype plants, we observed a dramatic decrease in Mg content in both roots and shoots of mgt6 mutants grown under low (0.01 mM) $\mathrm{Mg}$ conditions (Figure 4A). In the presence of normal $(1.5 \mathrm{mM})$ and high $(6 \mathrm{mM})$ external $\mathrm{Mg}^{2+}$ levels, mgt6 mutants also contained less $\mathrm{Mg}$ in both roots and shoots than wild-type plants, when the seedlings were grown on the plates (Figure 4A). Because $\mathrm{Ca}$ is usually associated with $\mathrm{Mg}$ homeostasis, we also measured $\mathrm{Ca}$ content in the plants. While $\mathrm{Ca}$ content in the root of mgt6 mutant was slightly higher, we surprisingly found that $\mathrm{Mg}$ deficiency resulted in a drastic reduction in shoot $\mathrm{Ca}$ compared with wild-type (Figure 4B). The Ca content, like other 

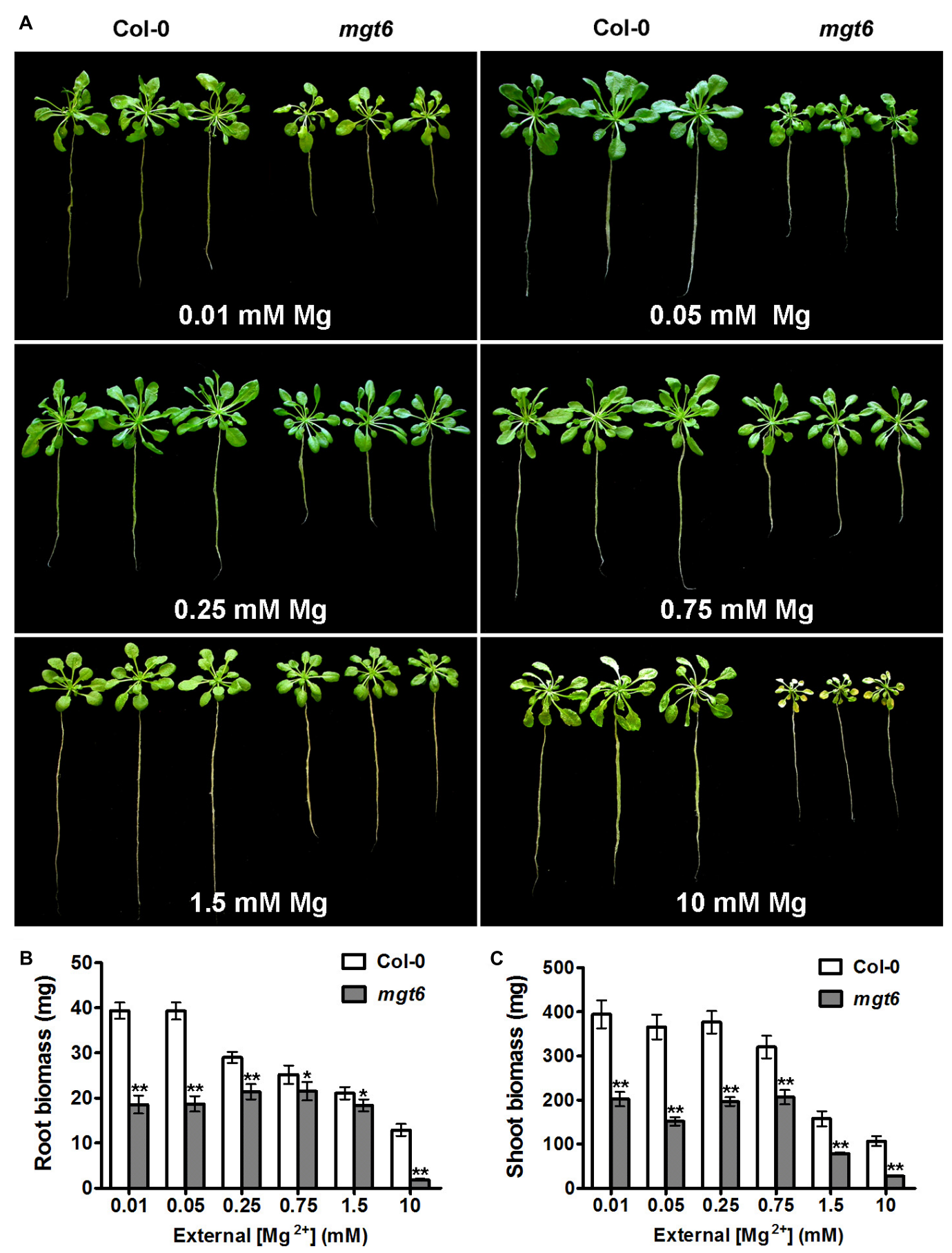

FIGURE 3 | Mature plant phenotypes of mgt6 mutants in a range of different external $\mathrm{Mg}^{2+}$ concentrations. (A) Growth phenotypes of 1-month-old wild-type plants and mgt6 mutants under hydroponic conditions containing indicated concentrations of $\mathrm{Mg}^{2+}$. (B) Quantification of root biomass. (C) Quantification of shoot biomass. Data represent means \pm SE of three replicate experiments. Asterisks indicate significant difference between the wild type and mgt6 mutant (Student's t-test, $* P<0.05, * * P<0.01)$

parameters of plant growth, was comparable between the wildtype and mutant plants grown under $1.5 \mathrm{mM} \mathrm{Mg}^{2+}$ (Figure 4B). The mgt6 mutant retained significantly less $\mathrm{Ca}$ in the root and slightly decreased Ca content in the shoot tissue when plants were cultured in $6 \mathrm{mM} \mathrm{Mg}^{2+}$ (Figure 4B).
We further measured the $\mathrm{Mg}$ and $\mathrm{Ca}$ content in the hydroponically grown mature plants. As the external $\mathrm{Mg}^{2+}$ levels increased, wild-type plants accumulated elevated amount of $\mathrm{Mg}$ in both root and shoot tissues. The mgt6 plants generally showed a significant reduction in root $\mathrm{Mg}$ content compared with 

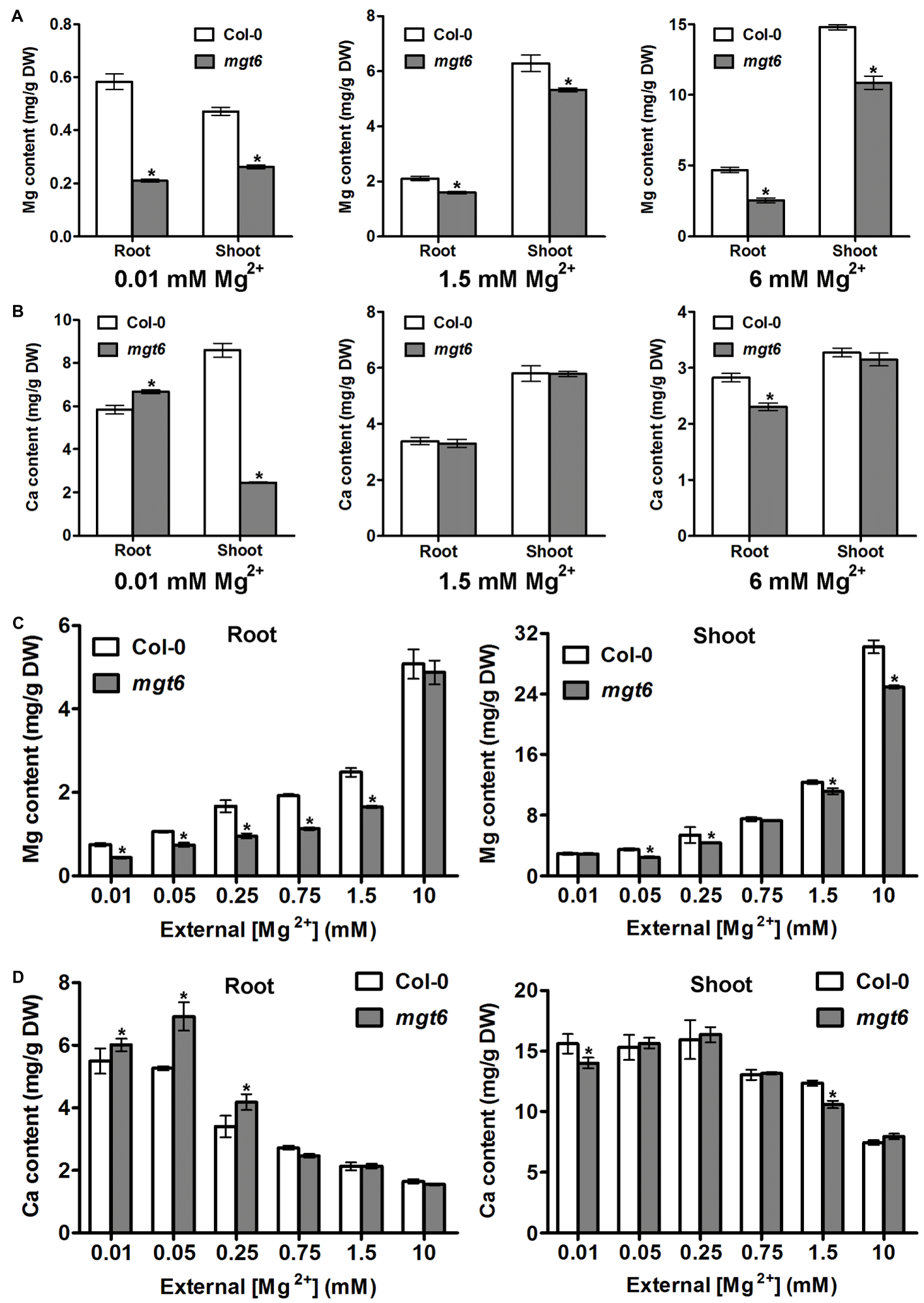

FIGURE 4 | Mg and Ca content in the mgt6 mutant under various growth conditions. (A) Mg content in the root and shoot of 2-week-old wild-type and mgt6 plants grown on the plates containing indicated concentrations of $\mathrm{Mg}^{2+}$. (B) Ca content in the root and shoot of 2-week-old wild-type and $m g t 6$ plants grown on the plates containing indicated concentrations of $\mathrm{Mg}^{2+}$. (C) $\mathrm{Mg}$ content in the root and shoot of 4-week-old wild-type and mgt6 plants grown in the hydroponic solutions containing various concentrations of $\mathrm{Mg}^{2+}$. (D) Ca content in the root and shoot of 4-week-old wild-type and mgt6 plants grown in the hydroponic solutions containing various concentrations of $\mathrm{Mg}^{2+}$. Data represent means $\pm \mathrm{SE}$ of four replicate experiments. Asterisks indicate significant difference between the wild type and mgt6 mutant (Student's $t$-test, $\left.{ }^{*} P<0.05\right)$. 
wild type, except under $10 \mathrm{mM} \mathrm{Mg}{ }^{2+}$ (Figure 4C). However, the shoot $\mathrm{Mg}$ content between wild type and mgt6 is most strikingly different under $10 \mathrm{mM} \mathrm{Mg}^{2+}$, although under some other concentrations of $\mathrm{Mg}^{2+}$, such as $0.05,0.25$, and $1.5 \mathrm{mM}$, mgt6 mutant also contained lower $\mathrm{Mg}$ content in the shoot compared with wild type (Figure 4C). Plant Ca contents are negatively correlated with external $\mathrm{Mg}^{2+}$ levels. Under low-Mg conditions $(0.01,0.05$, and $0.25 \mathrm{mM})$, an obvious elevation in root Ca was observed in the mgt6 mutant (Figure 4D), presumably due to the antagonistic interaction between $\mathrm{Mg}$ and $\mathrm{Ca}$. These results suggest MGT6 regulates plant $\mathrm{Mg}^{2+}$ homeostasis in both roots and shoots, and functions in a wide range of external $\mathrm{Mg}^{2+}$ concentrations at all developmental stages.

\section{Grafting Assay Uncovers a Shoot-Based Mechanism for MGT6 Function in High-Mg Tolerance}

While the low-Mg sensitive phenotype of $m g t 6$ can be explained by impaired $\mathrm{Mg}^{2+}$ uptake by root under $\mathrm{Mg}$-limited conditions in the mutant, the high-Mg susceptibility of mgt6 remains obscure. Since MGT6 controls both root and shoot $\mathrm{Mg}^{2+}$ homeostasis, we attempted to further investigate the mechanism by which MGT6 contributes to plant $\mathrm{Mg}^{2+}$ tolerance. Because MGT6 is widely expressed in plants, we decided to examine the relative contribution of MGT6 in roots versus in shoots through reciprocal grafting experiments between $m g t 6$ mutants and wildtype plants (Figure 5). When grown under low- $\mathrm{Mg}^{2+}$ conditions $(0.01 \mathrm{mM})$, the shoots with wild-type scions and $m g t 6$ rootstocks appeared to be smaller than that of self-grafted wild-type plants, although the root looked similar. The grafted plants with mgt6 scions and wild-type rootstocks were significantly smaller than wild-type self-grafted plants, but generally larger than mgt6 selfgrafted plants. Under the moderate level of $\mathrm{Mg}^{2+}(1.5 \mathrm{mM})$, both groups of the reciprocal grafted plants grew smaller than wild-type self-grafted plants. However, in the hydroponic culture containing $10 \mathrm{mM} \mathrm{Mg}{ }^{2+}$, which is considered to be a toxic concentration, the grafted plants with mgt6 scions and wildtype rootstocks phenocopied the defects seen in the mgt6 selfgrafts, whereas the grafted plants with wild-type scions and mgt6 rootstocks resembled the phenotype of wild-type selfgrafted plants (Figure 5A). We measured root and shoot fresh weight quantitatively, which verified the growth phenotypes (Figures 5B,C). These observations suggested that MGT6 is important in both root and shoot tissues when external $\mathrm{Mg}^{2+}$ is low and moderate. Presumably, MGT6-mediated absorption of external $\mathrm{Mg}^{2+}$ represents the dominant role under these conditions. When the external $\mathrm{Mg}^{2+}$ is extremely high, MGT6 function in the shoot becomes critical to detoxify excessive $\mathrm{Mg}^{2+}$ at the whole plant level. Consistent with this notion, wild-type scions grafted on mgt6 rootstocks lead to significantly lower root $\mathrm{Mg}^{2+}$ content under 0.01 and $1.5 \mathrm{mM} \mathrm{Mg}^{2+}$ conditions (Figures 6A,B). In the presence of $10 \mathrm{mM}$ external $\mathrm{Mg}^{2+}$, shoots from mgt6 grafted onto wild-type rootstocks retained much less $\mathrm{Mg}^{2+}$ in the shoot, similar to that observed in self-grafted mgt6 plants (Figure 6C). This further supported the idea that MGT6 fulfills a shoot-based mechanism to detoxify excessive
$\mathrm{Mg}^{2+}$, which could involve vacuolar $\mathrm{Mg}^{2+}$ storage based on the observation of lower $\mathrm{Mg}$ content in the mutant shoots.

\section{Functional Synergy of MGT6 and MGT7 in Arabidopsis}

Arabidopsis MGT7 encodes a low-affinity $\mathrm{Mg}^{2+}$ transporter (Mao et al., 2008) and is indispensable for optimal plant growth under low- $\mathrm{Mg}^{2+}$ conditions (Gebert et al., 2009). To investigate the functional interaction between MGT6 and MGT7, we created a double mutant that lacks both MGT6 and MGT7 transcripts (Supplementary Figure S3A). We found that the mgt6 mgt7 double mutant displayed pronounced growth retardation in the soil (Supplementary Figure S3B). Quantitative analysis indicated that the shoot fresh weight of the double mutant was only half of that of the wild type and single mutants (Supplementary Figure S3C).

We examined the growth phenotype of mgt6 mgt7 double mutant under various external $\mathrm{Mg}^{2+}$ concentrations, in comparison with wild-type as well as the mgt6 and mgt7 single mutants. While mgt6 single mutants exhibited very strong growth defects under both low- and high-Mg conditions, the phenotype of $m g t 7$ single mutant under the same condition was mild (Figure 7A). However, the $m g t 6 m g t 7$ double mutant was significantly more sensitive to external $\mathrm{Mg}^{2+}$ than the mgt6 single mutant (Figure 7A). The primary root of $m g t 6 m g t 7$ was shorter than that of mgt6 under low-Mg conditions (Figure 7B), although seedling fresh weight was comparable (Figure 7C). The leaf chlorophyll content in mgt6 mgt7was lower compared with mgt6 when high $\mathrm{Mg}^{2+}$ is present in the medium (Figure 7D).

Gene expression analysis indicated that a handful of gene markers (Kamiya et al., 2012) were more responsive to $\mathrm{Mg}$ starvation in the mgt6 or mgt7 mutant background than in the wild type, suggesting that the mgt6 and mgt7 mutants are impaired in low- $\mathrm{Mg}^{2+}$ adaptation. Consistent with the more severe phenotype, the mgt6 mgt7 double mutant displayed enhanced expression of Mg-starvation marker genes compared with the single mutants (Supplementary Figure S4). Taken together, these results indicate both MGT6 and MGT7 are important for plant $\mathrm{Mg}$ homeostasis and their functions are additive in regulating $\mathrm{Mg}^{2+}$ transport under a wide range of external $\mathrm{Mg}^{2+}$ concentrations.

\section{DISCUSSIONS}

In addition to air and water, plant growth and development rely on mineral nutrients taken up by roots and translocated into the shoot tissues through apoplast and symplast pathways, which entail not only transpiration-driven mass flow but also active membrane transport processes facilitated by various ion channels and transporters. $\mathrm{Mg}$ is an essential macronutrient in plants with diverse biological functions. However, the molecular mechanisms for $\mathrm{Mg}$ transport and homeostasis in plant cells remain largely unknown. Genomes of many plants such as Arabidopsis, rice, and maize, encode homologs of the bacterial CorA-type proteins referred to as MGTs/MRS2s (Li et al., 2001, 2016; Saito et al., 2013). Some members of the MGT family have been functionally 
A
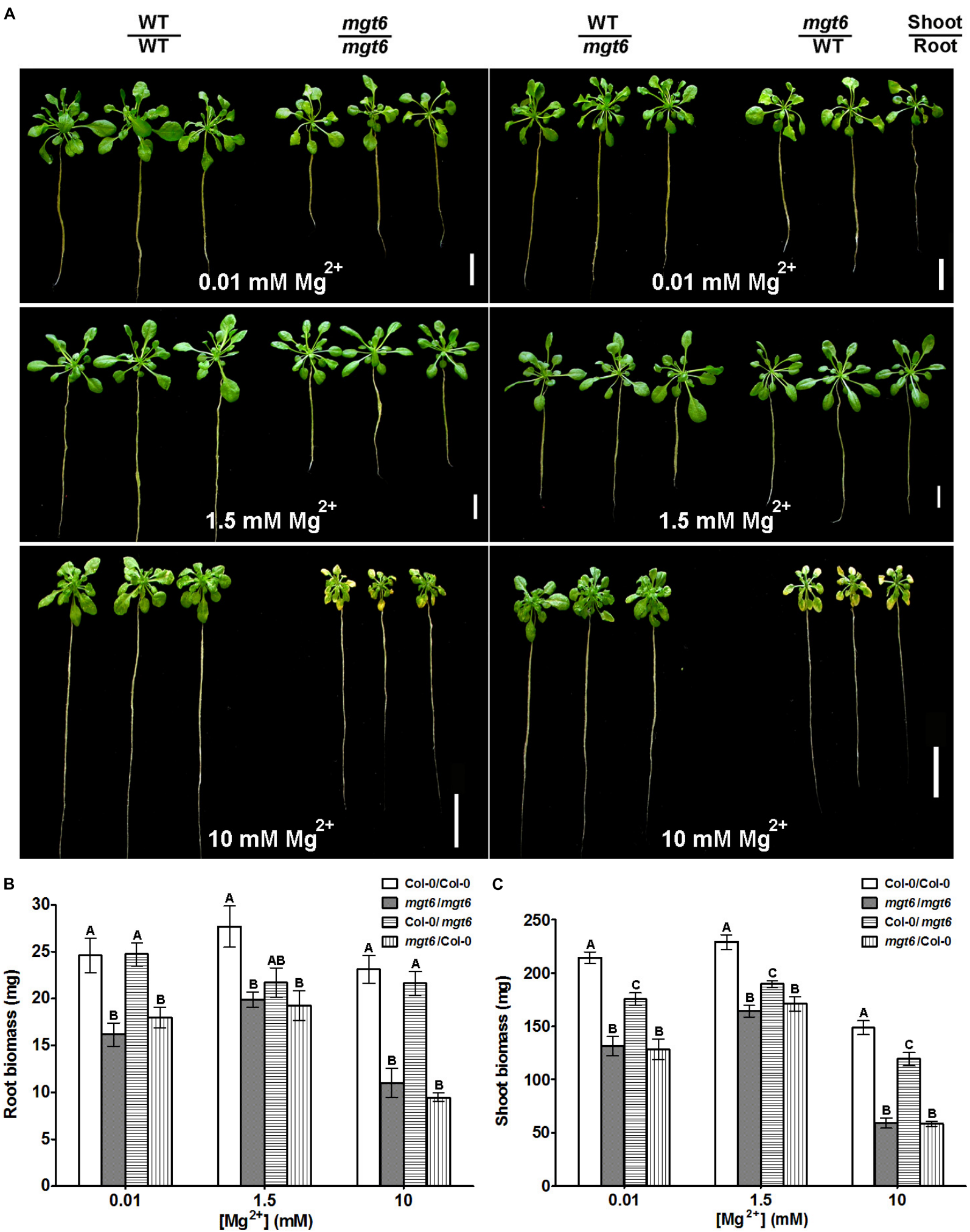

FIGURE 5 | Phenotypic analysis of reciprocal grafting of wild-type and mgt6 plants. (A) Growth phenotypes of 1-month-old grafted plants with different combinations under hydroponic conditions containing indicated concentrations of $\mathrm{Mg}^{2+}$. The genotype of the shoot scion is indicated in the upper part and the genotype of the rootstock is indicated in the lower part. Scale bar $=2 \mathrm{~cm}$. (B) Quantification of root biomass. (C) Quantification of shoot biomass. Data represent means \pm SE of three replicate experiments. Columns with different letters indicate significant difference (one-way ANOVA test, $P<0.05$ ). 

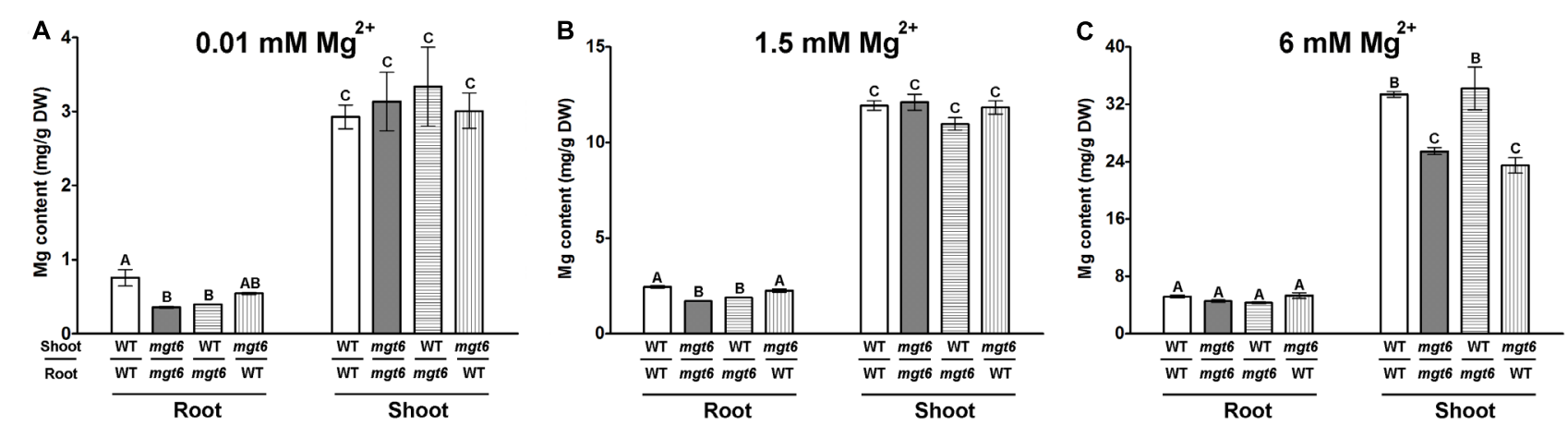

FIGURE 6 | Mg content in grafted plants under different external Mg $^{2+}$ conditions. (A-C) Determination of Mg content in the root and shoot tissues of four types of grafted plants grown in the hydroponic solutions containing different concentrations of $\mathrm{Mg}^{2+}(0.01,1.5$, and $6 \mathrm{mM})$. Data represent means $\pm \mathrm{SE}$ of four replicate experiments. Columns with different letters indicate significant difference (one-way ANOVA test, $P<0.05$ ).

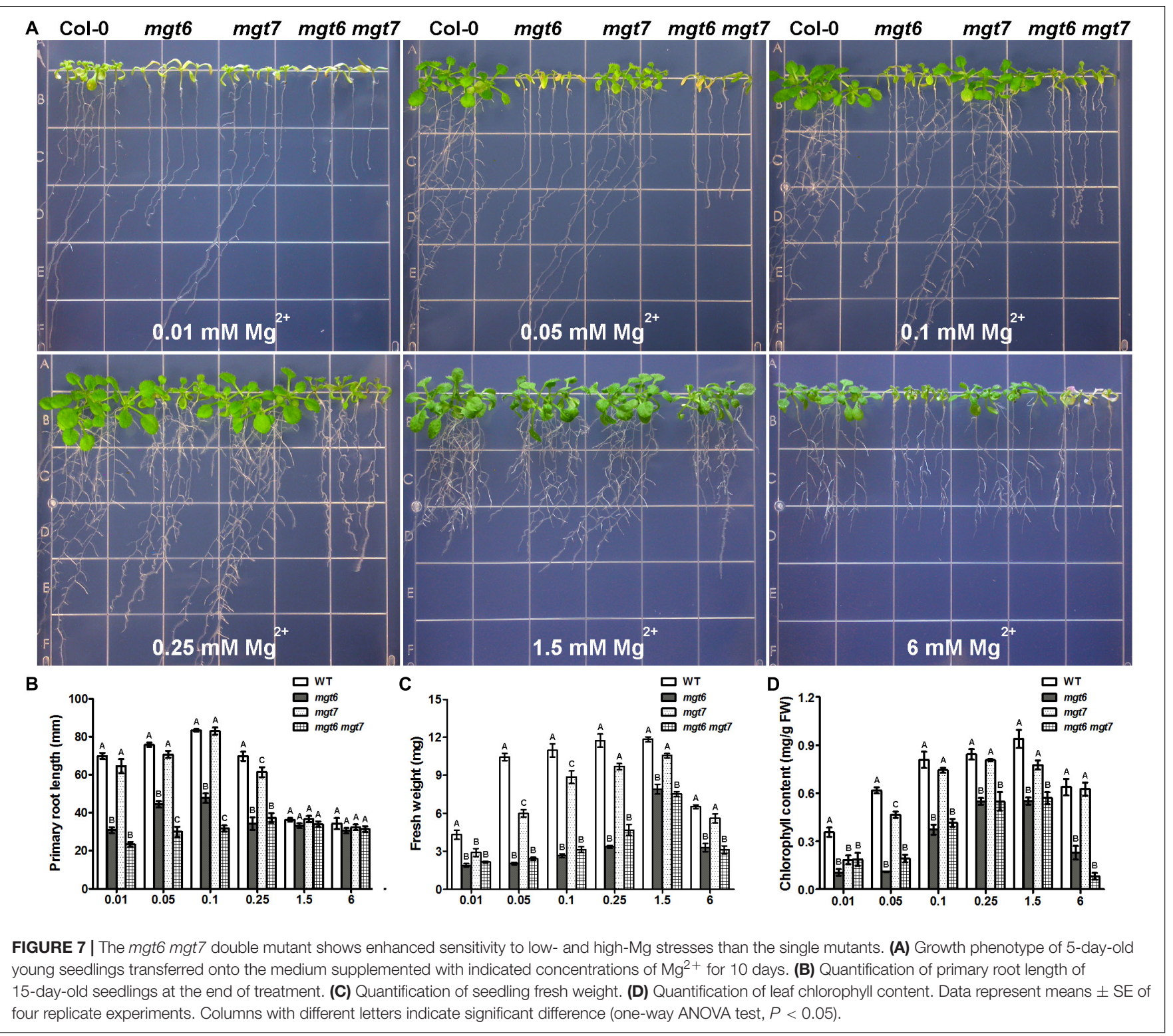


characterized, but the physiological roles of these transporters are not well understood. In Arabidopsis, we previously showed that MGT6 is capable of facilitating high-affinity $\mathrm{Mg}^{2+}$ uptake from the soil when external $\mathrm{Mg}^{2+}$ concentration is in the submillimolar range (Mao et al., 2014). Consistent with this role, expression of the MGT6 gene is highly inducible in the root tissues in response to low $\mathrm{Mg}$ (Mao et al., 2014). In the present study, we not only corroborated earlier findings regarding the critical role of MGT6 in low-Mg adaptation, but also extended the function of MGT6 in controlling plant $\mathrm{Mg}^{2+}$ homeostasis within a wide range of external $\mathrm{Mg}^{2+}$ levels. The $m g t 6$ knock-out mutant displayed obvious phenotype under high-Mg conditions, suggesting MGT6 exerts physiological functions in plants other than $\mathrm{Mg}^{2+}$ absorption. In higher plants, after absorption from the soil solution by roots, $\mathrm{Mg}^{2+}$ is believed to be transported to the aerial parts via transpiration stream moving through the xylem vessels. However, little is known about the molecular identity of the transporters involved in this long-distance transport. MGT6 might fulfill such a role in the xylem transport of $\mathrm{Mg}^{2+}$. Considering the negative membrane potential, $\mathrm{Mg}^{2+}$ is expected to be loaded passively into the pericycle cells. MGT6 may be responsible for $\mathrm{Mg}^{2+}$ import into pericycle and xylem parenchyma cells. On the other hand, the possibility that MGT6 serves as an "exporter" in this process cannot be excluded. It is generally believed that ion secretion occurs across plasmalemma of the parenchyma cells surrounding the xylem vessels (Clarkson, 1993; Gaymard et al., 1998). Interestingly, MGT5, the closest homolog of MGT6 in Arabidopsis, was shown to be a bidirectional $\mathrm{Mg}^{2+}$ carrier that operates in a concentration-dependent manner ( $\mathrm{Li}$ et al., 2008). Therefore, it is possible that MGT6 might also function in $\mathrm{Mg}^{2+}$ efflux from xylem parenchyma cells, pushing $\mathrm{Mg}^{2+}$ influx into the xylem vessel. Future electrophysiological analysis of MGT6 conductance is required to test this hypothesis.

Although $\mathrm{Mg}^{2+}$ is an essential mineral, high levels of $\mathrm{Mg}^{2+}$, such as those in the serpentine soils, could be toxic to plants. Recently we established that vacuolar sequestering of $\mathrm{Mg}^{2+}$, regulated by the tonoplast $\mathrm{CBL}-\mathrm{CIPK}$ signaling network, is crucial for plants to survive under high-Mg conditions (Tang et al., 2015). In the present work, we uncovered another component mainly fulfilled by MGT6 that underlies high-Mg tolerance at the whole plant level. Previous studies indicate that serpentine-adapted plants appear to efficiently transport $\mathrm{Mg}^{2+}$ from root to shoot, whereas the serpentine-sensitive counterparts are less capable of driving $\mathrm{Mg}^{2+}$ entry into the transpiration stream, resulting in a lower $\mathrm{Mg}^{2+}$ concentration in the shoot (Palm et al., 2012). Consistent with this finding, our physiological analysis of mgt6 mutant under high-Mg conditions showed that mgt6 retained considerably less $\mathrm{Mg}^{2+}$ in the shoot tissue compared to wild-type, accompanied by the growth retardation upon high-Mg stress. These data support the notion that long-distance $\mathrm{Mg}^{2+}$ transport mediated by MGT6 may play a critical role in protecting plants from $\mathrm{Mg}^{2+}$ toxicity at the whole plant level. More importantly, reciprocal grafting test indicated that MGT6 function in the shoot tissue is responsible for the high- $\mathrm{Mg}^{2+}$ tolerance. Considering the plasma membrane localization of MGT6, it is reasonable to speculate that MGT6 probably facilitates $\mathrm{Mg}^{2+}$ entry into the cytosol of leaf cells after the $\mathrm{Mg}^{2+}$ ions in the xylem unload into the apoplastic space. The excessive $\mathrm{Mg}^{2+}$ in the cytosol is subsequently sequestered into the central vacuole via tonoplastlocalized $\mathrm{Mg}^{2+}$ transporters. This transport cascade is critical for detoxification of excessive $\mathrm{Mg}^{2+}$, which is reminiscent of a recent model proposed for $\mathrm{Ca}^{2+}$ detoxification in plants (Wang et al., 2017).

Another notable finding in this study is that another MGTtype transporter MGT7 partially overlaps with the function of MGT6. With a preferential expression in the root, MGT7 was shown to be important for plant adaptation to lowMg conditions (Gebert et al., 2009). In our study, we found that although MGT6 plays a more dominant role in low-Mg conditions, MGT7 seems to be additive to MGT6 function because mgt6 mgt7 double mutant is more sensitive to low-Mg stress than the mgt6 single mutant, which is also supported by the enhanced activation of Mg-starvation gene expression in the double mutant. Interestingly, under high-Mg conditions, mutation of MGT7 also significantly enhanced the sensitivity of mgt6, although single mutant of mgt7 only exhibited a subtle phenotype under the same condition. These results suggest that MGT7 synergistically works together with MGT6 in the context of $\mathrm{Mg}^{2+}$ homeostasis at the whole plant level. Further investigations will sort out the mode of action for each of them to explain this functional synergy.

Subcellular localization of the MGT proteins may prove to be difficult to study. For instance, recent studies reported discrepant cellular localizations for MGT6 in the plasma membrane (Mao et al., 2014) and endoplasmic reticulum (ER; Oda et al., 2016), respectively. Several other MGT members such as MGT7 (Gebert et al., 2009) and MGT4 (Li et al., 2015) were also shown to be ER-associated, which needs to be re-evaluated because quite a few membrane proteins tend to be mis-targeted to ER, especially when overexpressed in a transient expression system (Denecke et al., 2012; Quattrocchio et al., 2013; Segami et al., 2014). Future studies using the native promoter, coupled with functional complementation in the mutant background as well as other approaches, are needed to verify the subcellular localization of MGT-type transporters in situ. It will also be interesting to examine if the targeting of MGT6 or MGT7 would be dynamically altered in different subcellular compartments in response to various $\mathrm{Mg}^{2+}$ concentrations.

As sessile organisms, plants have to cope with fluctuating concentrations of $\mathrm{Mg}^{2+}$ in nature. How plants maintain a balanced level of $\mathrm{Mg}^{2+}$ is not well understood. The present study as well as our previous work provides a working model in which MGT6 plays a dual role in controlling $\mathrm{Mg}^{2+}$ homeostasis. When external $\mathrm{Mg}^{2+}$ is limited, expression of MGT6 is induced in root epidermal cells and root hairs, making this transporter primarily responsible for $\mathrm{Mg}^{2+}$ uptake from the soil. When external $\mathrm{Mg}^{2+}$ is sufficient or becomes excessive, MGT6 mediates $\mathrm{Mg}^{2+}$ loading into the shoot tissues, where leaf mesophyll cells can subsequently sequester extra amount of $\mathrm{Mg}^{2+}$ into large vacuoles via yet-unknown transporters. Future efforts 
should be made in identifying uncharacterized $\mathrm{Mg}^{2+}$ transport proteins in plants. Furthermore, establishing the regulators and signaling pathways that fine-tune the expression and function of these transport systems will be a challenging but urgent task, which will ultimately lead to genetic manipulation of plants for precise adaption to the changing $\mathrm{Mg}^{2+}$ concentrations in the environment.

\section{AUTHOR CONTRIBUTIONS}

Y-WY and R-JT designed and conducted most of the experiments, interpreted the results, and wrote the draft of the manuscript. D-DM, X-XZ, Q-LT, and Y-PL assisted in some experiments and helped analyze the data. LY and J-LQ provided tools and reagents and made helpful discussions. SL supervised and conceptualized the study and finalized

\section{REFERENCES}

Brady, K. U., Kruckeberg, A. R., and Bradshaw, H. D. (2005). Evolutionary ecology of plant adaptation to serpentine soils. Annu. Rev. Ecol. Evol. Syst. 36, 243-266. doi: 10.1146/annurev.ecolsys.35.021103.105730

Chen, J., Li, L. G., Liu, Z. H., Yuan, Y. J., Guo, L. L., Mao, D. D., et al. (2009). Magnesium transporter AtMGT9 is essential for pollen development in Arabidopsis. Cell Res. 19, 887-898. doi: 10.1038/cr.2009.58

Chen, Z. C., Yamaji, N., Horie, T., Che, J., Li, J., An, G., et al. (2017). A Magnesium transporter OsMGT1 plays a critical role in salt tolerance in rice. Plant Physiol. 174, 1837-1849. doi: 10.1104/pp.17.00532

Chen, Z. C., Yamaji, N., Motoyama, R., Nagamura, Y., and Ma, J. F. (2012). Up-regulation of a magnesium transporter gene OsMGT1 is required for conferring aluminum tolerance in rice. Plant Physiol. 159, 1624-1633. doi: 10.1104/pp.112.199778

Clarkson, D. T. (1993). Roots and the delivery of solutes to the xylem. Philos. Trans. R. Soc. B 341, 5-17. doi: 10.1098/rstb.1993.0086

Clough, S. J., and Bent, A. F. (1998). Floral dip: a simplified method for Agrobacterium-mediated transformation of Arabidopsis thaliana. Plant J. 16, 735-743. doi: 10.1046/j.1365-313x.1998.00343.x

Conn, S. J., Conn, V., Tyerman, S. D., Kaiser, B. N., Leigh, R. A., and Gilliham, M. (2011). Magnesium transporters, MGT2/MRS2-1 and MGT3/MRS2-5, are important for magnesium partitioning within Arabidopsis thaliana mesophyll vacuoles. New Phytol. 190, 583-594. doi: 10.1111/j.1469-8137.2010. 03619.x

Denecke, J., Aniento, F., Frigerio, L., Hawes, C., Hwang, I., Mathur, J., et al. (2012). Secretory pathway research: the more experimental systems the better. Plant Cell 24, 1316-1326. doi: 10.1105/tpc.112.096362

Drummond, R. S. M., Tutone, A., Li, Y. C., and Gardner, R. C. (2006). A putative magnesium transporter AtMRS2-11 is localized to the plant chloroplast envelope membrane system. Plant Sci. 170, 78-89. doi: 10.1016/j.plantsci.2005. 08.018

Eshaghi, S., Niegowski, D., Kohl, A., Molina, D. M., Lesley, S. A., and Nordlund, P. (2006). Crystal structure of a divalent metal ion transporter CorA at 2.9 angstrom resolution. Science 313, 354-357. doi: 10.1126/science.1127121

Gaymard, F., Pilot, G., Lacombe, B., Bouchez, D., Bruneau, D., Boucherez, J., et al. (1998). Identification and disruption of a plant shaker-like outward channel involved in $\mathrm{K}+$ release into the xylem sap. Cell 94, 647-655. doi: 10.1016/S00928674(00)81606-2

Gebert, M., Meschenmoser, K., Svidova, S., Weghuber, J., Schweyen, R., Eifler, K., et al. (2009). A root-expressed magnesium transporter of the MRS2/MGT gene family in Arabidopsis thaliana allows for growth in low-Mg2+ environments. Plant Cell 21, 4018-4030. doi: 10.1105/tpc.109.070557

Hermans, C., Conn, S. J., Chen, J. G., Xiao, Q. Y., and Verbruggen, N. (2013). An update on magnesium homeostasis mechanisms in plants. Metallomics 5, 1170-1183. doi: 10.1039/c3mt20223b the paper. All the authors approved the final version of the manuscript.

\section{FUNDING}

This work was funded by National Science Foundation to SL and National Natural Science Foundation of China (31500200) to D-DM. Y-WY was in part supported by a fellowship from the China Scholarship Council.

\section{SUPPLEMENTARY MATERIAL}

The Supplementary Material for this article can be found online at: https://www.frontiersin.org/articles/10.3389/fpls.2018.00274/ full\#supplementary-material

Kamiya, T., Yamagami, M., Hirai, M. Y., and Fujiwara, T. (2012). Establishment of an in planta magnesium monitoring system using $C A X 3$ promoter-luciferase in Arabidopsis. J. Exp. Bot. 63, 355-363. doi: 10.1093/jxb/err283

Li, H. Y., Du, H. M., Huang, K. F., Chen, X., Liu, T. Y., Gao, S. B., et al. (2016). Identification, and functional and expression analyses of the CorA/MRS2/MGT-Type magnesium transporter family in maize. Plant Cell Physiol. 57, 1153-1168. doi: 10.1093/pcp/pcw064

Li, J., Huang, Y., Tan, H., Yang, X., Tian, L., Luan, S., et al. (2015). An endoplasmic reticulum magnesium transporter is essential for pollen development in Arabidopsis. Plant Sci. 231, 212-220. doi: 10.1016/j.plantsci.2014.12.008

Li, L., Tutone, A. F., Drummond, R. S., Gardner, R. C., and Luan, S. (2001). A novel family of magnesium transport genes in Arabidopsis. Plant Cell 13, 2761-2775. doi: $10.1105 /$ tpc.13.12.2761

Li, L. G., Sokolov, L. N., Yang, Y. H., Li, D. P., Ting, J., Pandy, G. K., et al. (2008). A mitochondrial magnesium transporter functions in Arabidopsis pollen development. Mol. Plant 1, 675-685. doi: 10.1093/mp/ssn031

Liang, S., Qi, Y. F., Zhao, J., Li, Y. F., Wang, R., Shao, J. X., et al. (2017). Mutations in the Arabidopsis AtMRS2-11/AtMGT10/VAR5 gene cause leaf reticulation. Front. Plant Sci. 8:2007. doi: 10.3389/fpls.2017.02007

Lunin, V. V., Dobrovetsky, E., Khutoreskaya, G., Zhang, R., Joachimiak, A., Doyle, D. A., et al. (2006). Crystal structure of the CorA Mg2+ transporter. Nature 440, 833-837. doi: 10.1038/nature04642

Mao, D., Chen, J., Tian, L., Liu, Z., Yang, L., Tang, R., et al. (2014). Arabidopsis transporter MGT6 mediates magnesium uptake and is required for growth under magnesium limitation. Plant Cell 26, 2234-2248. doi: 10.1105/tpc.114. 124628

Mao, D. D., Tian, L. F., Li, L. G., Chen, J., Deng, P. Y., Li, D. P., et al. (2008). AtMGT7: an Arabidopsis gene encoding a low-affinity magnesium transporter. J. Integr. Plant Biol. 50, 1530-1538. doi: 10.1111/j.1744-7909.2008.00770.x

Marsch-Martínez, N., Franken, J., Gonzalez-Aguilera, K. L., De Folter, S., Angenent, G., and Alvarez-Buylla, E. R. (2013). An efficient flat-surface collarfree grafting method for Arabidopsis thaliana seedlings. Plant Methods 9:14. doi: 10.1186/1746-4811-9-14

Murashige, T., and Skoog, F. (1962). A revised medium for rapid growth and bioassays with tobacco tissue cultures. Physiol. Plant 15, 473-495. doi: 10.1111/ j.1399-3054.1962.tb08052.x

Oda, K., Kamiya, T., Shikanai, Y., Shigenobu, S., Yamaguchi, K., and Fujiwara, T. (2016). The Arabidopsis Mg transporter, MRS2-4, is essential for $\mathrm{Mg}$ homeostasis under both low and high Mg conditions. Plant Cell Physiol. 57, 754-763. doi: 10.1093/pcp/pcv196

Palm, E., Brady, K., and Van Volkenburgh, E. V. (2012). Serpentine tolerance in Mimulus guttatus does not rely on exclusion of magnesium. Funct. Plant Biol. 39, 679-688. doi: 10.1007/s00442-009-1448-0

Quattrocchio, F. M., Spelt, C., and Koes, R. (2013). Transgenes and protein localization: myths and legends. Trends Plant Sci. 18, 473-476. doi: 10.1016/j. tplants.2013.07.003 
Saito, T., Kobayashi, N. I., Tanoi, K., Iwata, N., Suzuki, H., Iwata, R., et al. (2013). Expression and functional analysis of the CorA-MRS2-ALR-type magnesium transporter family in rice. Plant Cell Physiol. 54, 1673-1683. doi: 10.1093/pcp/ pct112

Schock, I., Gregan, J., Steinhauser, S., Schweyen, R., Brennicke, A., and Knoop, V. (2000). A member of a novel Arabidopsis thaliana gene family of candidate $\mathrm{Mg} 2+$ ion transporters complements a yeast mitochondrial group II intronsplicing mutant. Plant J. 24, 489-501. doi: 10.1046/j.1365-313x.2000.00895.x

Segami, S., Makino, S., Miyake, A., Asaoka, M., and Maeshima, M. (2014). Dynamics of vacuoles and $\mathrm{H}^{+}$-pyrophosphatase visualized by monomeric green fluorescent protein in Arabidopsis: artifactual bulbs and native intravacuolar spherical structures. Plant Cell 26, 3416-3434. doi: 10.1105/tpc. 114.127571

Shaul, O. (2002). Magnesium transport and function in plants: the tip of the iceberg. Biometals 15, 309-323. doi: 10.1023/A:1016091118585

Sun, Y., Yang, R. A., Li, L. G., and Huang, J. R. (2017). The magnesium transporter MGT10 is essential for chloroplast development and photosynthesis in Arabidopsis thaliana. Mol. Plant 10, 1584-1587. doi: 10.1016/j.molp.2017.09.017

Szegedy, M. A., and Maguire, M. E. (1999). The CorA $\mathrm{Mg}^{2+}$ transport protein of Salmonella typhimurium mutagenesis of conserved residues in the second membrane domain. J. Biol. Chem. 274, 36973-36979. doi: 10.1074/jbc.274.52. 36973

Tang, R. J., and Luan, S. (2017). Regulation of calcium and magnesium homeostasis in plants: from transporters to signaling network. Curr. Opin. Plant Biol. 39, 97-105. doi: 10.1016/j.pbi.2017.06.009
Tang, R. J., Zhao, F. G., Garcia, V. J., Kleist, T. J., Yang, L., Zhang, H. X., et al. (2015). Tonoplast CBL-CIPK calcium signaling network regulates magnesium homeostasis in Arabidopsis. Proc. Natl. Acad. Sci. U.S.A. 112, 3134-3139. doi: $10.1073 /$ pnas. 1420944112

Turner, T. L., Bourne, E. C., Von Wettberg, E. J., Hu, T. T., and Nuzhdin, S. V. (2010). Population resequencing reveals local adaptation of Arabidopsis lyrata to serpentine soils. Nat. Genet. 42, 260-263. doi: 10.1038/ng.515

Wang, Y., Kang, Y., Ma, C., Miao, R., Wu, C., Long, Y., et al. (2017). CNGC2 is a $\mathrm{Ca} 2+$ influx channel that prevents accumulation of apoplastic $\mathrm{Ca} 2+$ in the leaf. Plant Physiol. 173, 1342-1354. doi: 10.1104/pp.16.01222

Xu, X. F., Wang, B., Lou, Y., Han, W. J., Lu, J. Y., Li, D. D., et al. (2015). Magnesium transporter 5 plays an important role in Mg transport for male gametophyte development in Arabidopsis. Plant J. 84, 925-936. doi: 10.1111/tpj. 13054

Conflict of Interest Statement: The authors declare that the research was conducted in the absence of any commercial or financial relationships that could be construed as a potential conflict of interest.

Copyright $\odot 2018$ Yan, Mao, Yang, Qi, Zhang, Tang, Li, Tang and Luan. This is an open-access article distributed under the terms of the Creative Commons Attribution License (CC BY). The use, distribution or reproduction in other forums is permitted, provided the original author(s) and the copyright owner are credited and that the original publication in this journal is cited, in accordance with accepted academic practice. No use, distribution or reproduction is permitted which does not comply with these terms. 